\title{
Estado, cooperativas y legislación cooperativa en la hora actual
}

\author{
Dante Cracogna \\ Universidad de Buenos Aires
}

Sumario: I. Introducción. II. Estado, cooperativas y desarrollo. III. Las cooperativas y la tradición jurídica nacional. IV. Relevancia de la legislación cooperativa. V. Definición de cooperativa. VI. Constitución y reconocimiento legal. VII. Los socios. VIII. Régimen económico. IX. Contabilidad y auditoría. X. Los órganos sociales. XI. Disolución y liquidación. XII. Organizaciones de integración y representación. XIII. La supervisión estatal y el autocontrol. XIV. La política nacional en materia de cooperativas. XV. Los conflictos en las cooperativas y su solución. XVI. Aspectos fiscales. XVII. Consideraciones finales.

Resumen: La relación entre Estado y cooperativas y la legislación cooperativa vuelve a estar en boga puesto que la Resolución que proclama el año 2012 como Año Internacional de las Cooperativas «alienta a los gobiernos a que sigan examinando, según proceda, las disposiciones jurídicas y administrativas que rigen las actividades de las cooperativas a fin de promover su crecimiento y sostenibilidad en un entorno socioeconómico que evoluciona con rapidez». Resulta, por lo tanto, oportuno reflexionar sobre algunos lineamientos básicos que la legislación cooperativa debería tomar en cuenta para su adecuación a las exigencias de la hora actual.

Palabras-clave: legislación cooperativa, cooperativismo, Estado.

Abstract: The relationship between State and cooperatives and cooperative legislation is back in fashion as the resolution that declares 2012 as International Year of Cooperatives «encourages governments to continue revising, as appropriate, the legal and administrative provisions governing the activities carried out by cooperatives to promote their growth and sustainability in a socioeconomic context that is rapidly changing». It is therefore appropriate to reflect on some basic guidelines that cooperative legislation should consider to adapt to current demands.

Key words: cooperative legislation, cooperativism, State. 


\section{Introducción}

La declaración del Año Internacional de las Cooperativas por parte de las Naciones Unidas brinda oportunidad propicia para volver sobre el tema de la relación entre Estado y cooperativas y la legislación cooperativa puesto que la Resolución que proclama el año 2012 como tal contiene varias referencias a esta cuestión ${ }^{1}$. En efecto, "alienta a los gobiernos a que sigan examinando, según proceda, las disposiciones jurídicas y administrativas que rigen las actividades de las cooperativas a fin de promover su crecimiento y sostenibilidad en un entorno socioeconómico que evoluciona con rapidez, entre otras coas, estableciendo para las cooperativas condiciones equiparables a las de otras empresas comerciales y sociales, incluidos incentivos fiscales apropiados y el acceso a los servicios y mercados financieros.» Asimismo insta a los gobiernos a que «presten la debida atención al papel y la contribución de las cooperativas» en la aplicación de los distintos pronunciamientos de encuentros internacionales promovidos por la ONU «tomando medidas apropiadas para crear un entorno propicio y favorable al desarroIlo de las cooperativas ... promoviendo e implementando mejor legislación...»

Resulta, por lo tanto, oportuno reflexionar sobre algunos lineamientos básicos que la legislación cooperativa debería tomar en cuenta para su adecuación a las exigencias de la hora actual.

\section{Estado, cooperativas y desarrollo}

El debate acerca de la relación entre el Estado y las cooperativas es una constante tanto en la teoría como en la práctica del movimiento cooperativo. Si bien el tema cobró especial auge en las décadas posteriores a la Segunda Guerra Mundial ya se había planteado mucho antes, especialmente con motivo de la acción que algunos los gobiernos coloniales llevaron a cabo en materia de cooperativas ${ }^{2}$. Mientras que en los países europeos, en general, la cuestión sólo se formulaba en términos del reconocimiento legal de las cooperativas por parte del Estado como organizaciones de una naturaleza peculiar, en los países de Asia, África y América Latina, la cuestión se planteaba en el marco de

1 Resolución 64/136 de la Asamblea General.

2 Hans-H. MÜNKNER, Introduction into the Seminar Concept of State-sponsored Cooperative, Report on the ACO-SNCF-FES Seminar on Cooperative Law in ASEAN, Singapore, 10-14 April 1984. 
la acción promocional que correspondía al Estado llevar adelante para impulsar el desarrollo económico y social del respectivo país ${ }^{3}$.

Por lo tanto, en el llamado Tercer Mundo el problema de la relación entre el Estado y las cooperativas se vinculó desde el inicio con el tema del desarrollo y la discusión se desplazó hacia el papel de las cooperativas como elementos o factores desarrollo. La temática pasó a ser, entonces, cómo se relacionan Estado y cooperativas para promover el desarrollo, lo cual llevó a considerar a las cooperativas como una suerte de colaboradores o auxiliares del Estado en la realización de esa actividad ${ }^{4}$.

Sin embargo, en los últimos años el tema ha experimentado un cambio sustancial provocado por la reconsideración de las cooperativas como organizaciones autónomas al servicio de las necesidades de sus socios, por una parte y, por otra, por los negativos resultados exhibidos por la experiencia de diferentes países en los que las cooperativas se habían convertido en poco menos que oficinas gubernamentales ${ }^{5}$.

De manera que en el contexto actual esta cuestión se presenta en términos diferentes dentro de los cuales se inscriben la Declaración de Identidad Cooperativa formulada por la Alianza Cooperativa Internacional en el Congreso de Manchester 19956, los Lineamientos para la Creación de un Contexto Favorable para el Desarrollo de las Cooperativas recomendados por las Naciones Unidas en $2001^{7}$ y la Recomendación 193 de la OIT sobre la promoción de las cooperativas de $2002^{8}$. Por otra parte, se ha extendido la consideración del tema a todos los países con carácter general, sean industrializados, en transición o en vías de desarrollo, produciéndose un cambio de enfoque, no limitado exclusivamente a estos últimos.

3 Dante CRACOGNA, The Relationship between the State and Cooperatives in Cooperative Development, Informe del Coloquio realizado en Ginebra, 15 diciembre 1993, ILO, Geneva, 1993.

4 Dieter W. BENECKE, Cooperación y Desarrollo, ICECOOP, Santiago de Chile, 1973, p. 277 y ss.

5 Hans-H. MÜNKNER, Rediscovery of Co-operatives in Development Policy, ICA ROAP Sub-Regional Workshop on Co-operative Policy Reforms, Kathmandu, 13-16 January 1999, Reprint from COOP Dialogue, An ICA ROAP Journal, Vol. 10, No 1, January 2000, pp. 8-13.

6 ICA Statement on the Cooperative Identity, Review of International Cooperation, Vol. 88, No 41985.

7 UN Document A/56/73-E/2001/68.

8 Significativamente, este documento vino a reemplazar a la anterior Recomendación 127 de 1966 que estaba referida únicamente a las cooperativas en los países en desarrollo. 
En suma, las cooperativas buscan autónomamente el mejoramiento económico de sus socios y al hacerlo promueven el bienestar y el desarrollo de sus comunidades. Dentro de ese contexto se inscribe actualmente el tratamiento de este tema.

\section{Las cooperativas y la tradición jurídica nacional}

Las cooperativas tienen una naturaleza universal toda vez que constituyen la expresión de valores que no reconocen fronteras; son una manifestación de la voluntad libre de las personas que procuran resolver sus necesidades mediante el esfuerzo propio y la ayuda mutua, es decir organizando la acción en común con quienes tienen las mismas necesidades y aspiraciones.

Este carácter universal de la cooperación se halla plasmado en la Declaración de Identidad Cooperativa de la $\mathrm{ACl}$ en la que, después de la definición de cooperativa, se enuncian los valores básicos sobre los que asientan estas organizaciones y luego los principios por medio de los cuales las cooperativas ponen en práctica esos valores. Tales instrumentos — definición, valores y principios - tienen alcance universal.

La cooperativa es concebida como asociación autónoma de personas que se unen voluntariamente para satisfacer necesidades y aspiraciones económicas sociales y culturales comunes por medio de una empresa de propiedad conjunta democráticamente gestionada. Así definida la cooperativa, sus valores básicos son autoayuda, autoresponsabilidad, democracia, igualdad, equidad y solidaridad, a los que se agregan los valores éticos que sostienen los socios de las cooperativas, a saber: honestidad, apertura, responsabilidad social y preocupación por los demás. La universalidad de ese plexo resulta evidente. Luego se establecen las pautas generales conforme con las cuales las cooperativas ponen en práctica dichos valores, es decir los principios cooperativos.

Frente a esa universalidad las cooperativas son, sin embargo, organizaciones que tienen un profundo arraigo local; es decir que nacen y se desarrollan en un determinado contexto histórico, geográfico y cultural, lo cual les confiere un perfil propio de su lugar y tiempo. Dentro de ese contexto local, un aspecto de singular especificidad es el sistema jurídico vigente, el cual generalmente está ligado a tradiciones histórico políticas particulares de cada país.

De lo dicho se desprende el interrogante acerca de cómo conciliar la naturaleza universal de la cooperación —sus valores y principios- 
con el carácter necesariamente local de las cooperativas. Este problema puede conducir a dos soluciones igualmente equivocadas: por una parte, pretender una legislación uniforme para los diferentes países con independencia de sus respectivas culturas y tradiciones jurídicas ${ }^{9} \mathrm{y}$, por otra, afirmar una legislación nacional que regule a las cooperativas con total prescindencia de las notas generales que definen y caracterizan a estas organizaciones. La síntesis correcta, en cambio, consiste en brindar a las cooperativas un tratamiento legal que tome en consideración sus rasgos de alcance universal pero con respeto del sistema jurídico nacional ${ }^{10}$. Obviamente, se trata de una solución compleja que requiere un adecuado conocimiento del tema para poder traducirlo en las normas legales pertinentes ${ }^{11}$.

\section{Relevancia de la legislación cooperativa}

La legislación cooperativa constituye la primera y fundamental manifestación de la actitud del Estado hacia las cooperativas; ella expresa cómo el Estado considera a estas organizaciones en cuanto a su naturaleza, su funcionamiento, sus actividades, etc. Si bien hay algunos países en los cuales no existe legislación específica sobre cooperativas ${ }^{12}$, se trata de casos excepcionales pues en la generalidad de los países existen disposiciones legales sobre cooperativas, sea que estén contenidas en cuerpos más amplios (tales como los códigos de comercio o civil) o se trate de leyes autónomas.

9 Esta solución se ha intentado mediante la transcripción de leyes de cooperativas pertenecientes a países donde las cooperativas alcanzaron un significativo desarrollo, como si con ello pudieran incorporarse al mismo tiempo las experiencias exitosas de los países de origen.

10 Para facilitar esta tarea se han elaborado leyes modelo que sirven de orientación para la tarea de los legisladores al proponer contenidos básicos relacionados con los rasgos universales de las cooperativas, dejando los demás aspectos para su definición conforme con las características del sistema jurídico de cada país. Ejemplo representativo es la Ley marco para las cooperativas de América Latina, elaborado por un grupo de expertos de la Organización de las Cooperativas de América (OCA) en 1988 y actualizado por la Alianza Cooperativa Internacional para las Américas en 2008 (Dante Cracogna, "Nueva versión de la Ley Marco para las cooperativas de América Latina», Revista Jurídica de Economía Social y Cooperativa, N. ${ }^{\circ} 20$, Valencia, 2009, p. 183 y ss).

11 Una excelente orientación para esta tarea brinda Hagen Henrÿ, Guidelines for Cooperative Legislation, 2nd. edition, ILO, Geneva, 2005, passim.

12 Son conocidos los casos de Dinamarca y Noruega, países que hasta épocas reciente no contaban con leyes específicas de cooperativas pero que, sin embargo, tienen un importante movimiento cooperativo. 
La existencia de una legislación especial sobre cooperativas — sea en forma autónoma o dentro de un cuerpo más amplio- constituye un requisito necesario para caracterizar a las cooperativas y brindarles un marco adecuado para su organización y desenvolvimiento. Esta legislación puede tener la forma de una ley general única para toda clase de cooperativas (a veces con capítulos específicos para los distintos tipos) o bien puede manifestarse en leyes separadas para las diferentes clases de cooperativa en particular.

Puede afirmarse que parece más conveniente la existencia de una sola ley general referida a toda clase de cooperativas, pues de esa manera se afirma el carácter único y común de todas ellas, aunque contenga disposiciones especiales para determinadas clases en particular. Por otra parte, la existencia de una ley general evita las posibles contradicciones o superposiciones que podrían producirse entre diferentes leyes. Es asimismo conveniente que sea una ley autónoma, es decir no integrante de otro cuerpo legislativo, pues de esa manera se afirma la naturaleza propia y distintiva de las cooperativas.

Sin embargo, debe tenerse presente que la legislación cooperativa no representa la totalidad de las disposiciones legales que rigen a esta entidades puesto que ellas se encuentran también sometidas a otras leyes que inciden sobre sus actividades. Por lo tanto, ha de cuidarse que por vía de estas últimas no queden las cooperativas sometidas a disposiciones que afecten negativamente su desenvolvimiento o puedan contrariar su naturaleza. Suele ocurrir que, aun contando con una adecuada legislación específica sobre cooperativas, existan disposiciones en otras leyes que traben o dificulten sus actividades.

Asimismo debe tenerse en cuenta que las reglamentaciones y resoluciones administrativas de rango inferior a la legislación deben ser consistentes con ésta para que no desvirtúen sus disposiciones. Para asegurar que ello no ocurra, o que pueda remediarse rápidamente si llegara a suceder, es necesario contar con mecanismos apropiados previstos en la misma legislación cooperativa. Por otra parte, es necesario que la ley contenga todos los aspectos fundamentales relativos a la regulación de las cooperativas, dejando a las reglamentaciones y resoluciones administrativas sólo aquéllos que sean de carácter secundario ${ }^{13}$.

13 Tanto el Anexo de la Resolución 56/114 de la ONU como la Recomendación 193 de la OIT contienen un resumen de las principales materias acerca de las cuales debería incluir disposiciones la legislación cooperativa. 


\section{Definición de cooperativa}

La definición legal de la cooperativa es una cuestión de fundamental importancia puesto que constituye el núcleo a partir del cual se desenvuelve la legislación cooperativa. La correcta definición de la cooperativa es, pues, la piedra de toque para una adecuada ley. La apropiada definición permite distinguir claramente a la cooperativa de otras formas de organización jurídica, tales como las sociedades comerciales, las asociaciones, etc. y así perfilar la naturaleza específica de aquélla.

La Declaración de Identidad Cooperativa de la $\mathrm{ACl}$ contiene una definición de cooperativa ${ }^{14}$ que puede servir de adecuada orientación, la cual se halla reproducida textualmente en la Recomendación 193 de la OIT. Sin embargo, en todo caso la definición debe adecuarse a las características propias del sistema jurídico nacional.

Los principios cooperativos deben asimismo tener acogida dentro de la caracterización de las cooperativas puesto que ellos contribuyen a precisar con más rigor la naturaleza de estas organizaciones. No obstante, debe tenerse en cuenta que, conforme lo señala la Declaración de Identidad Cooperativa, los principios son «pautas generales» por medio de las cuales las cooperativas ponen en práctica sus valores. Es decir que admiten adecuaciones, siempre que ellas no alteren su significado fundamental.

La apropiada recepción de la definición y de los principios cooperativos constituye un requisito fundamental de la legislación cooperativa para caracterizar a las genuinas cooperativas evitando confusiones o usos indebidos de la denominación. Ello debe complementarse con la prohibición del uso de tal nombre por parte de entidades que no encuadren dentro de las disposiciones legales respectivas.

\section{Constitución y reconocimiento legal}

La legislación cooperativa debe establecer el carácter de sujeto de derecho de la cooperativa, es decir su consideración como una entidad a la que el ordenamiento jurídico nacional le reconoce su existencia con todas prerrogativas que son propias de los entes de esta naturaleza, en

14 «Una cooperativa es una asociación autónoma de personas que se unen voluntariamente para satisfacer sus necesidades y aspiraciones económicas, sociales y culturales comunes por medio de una empresa de propiedad conjunta, democráticamente gestionada.» 
igualdad de condiciones con las sociedades comerciales, las asociaciones civiles y otras formas jurídicas admitidas por el derecho nacional ${ }^{15}$.

La ley debe establecer en forma clara y precisa el procedimiento para que la cooperativa adquiera su reconocimiento legal, el cual ha de ser similar al que esté previsto para las sociedades comerciales y las asociaciones, sin exigencias costosas ni requisitos excesivos ${ }^{16}$. Generalmente dicho procedimiento consistirá en la inscripción en un registro especial, que puede ser una sección del mismo que inscribe a las demás organizaciones, una vez cumplidas las formalidades respectivas. La denegatoria de la inscripción en el registro podrá ser recurrida ante un tribunal a fin de que pueda ejercerse un control judicial sobre las decisiones administrativas, evitando su arbitrariedad.

La exigencia de un estatuto que satisfaga los requerimientos impuestos por la ley y que contemple adecuadamente todos los principales aspectos relacionados con la organización, el funcionamiento y la liquidación de la cooperativa, es condición indispensable para otorgar la inscripción en el registro, puesto que ese documento libremente aceptado por los socios es el que regirá la vida de la cooperativa y su relación con los socios. La provisión de estatutos tipo o modelo por parte del registro o de las organizaciones cooperativas de grado superior puede ayudar eficazmente a la constitución de cooperativas, siempre que sólo sean de orientación y no obligatorios.

El registro debe ser público, es decir abierto a todos los que desean saber acerca de las organizaciones que se hallan inscriptas en él. Ello sin perjuicio de que se efectúen publicaciones que difundan las inscripciones que se realizan, además de otorgarse constancias a las cooperativas registradas.

\section{Los socios}

La adhesión a las cooperativas es una decisión libre y voluntaria, abierta a todas las personas capaces de utilizar sus servicios y que acep-

15 Se discute acerca de la conveniencia de las llamadas «pre-cooperativas» como organizaciones previas a la constitución legal de las cooperativas. En general, sería conveniente adecuar los requisitos para la constitución de las cooperativas en lugar de crear una nueva figura intermedia de perfil impreciso y que se presta a confusión en cuanto a su naturaleza.

16 La Recomendación 193 de la OIT puntualiza: «establecer un marco institucional que permita proceder al registro de las cooperativas de la manera más rápida, sencilla, económica y eficaz posible» (punto II, 6). 
ten las responsabilidades de asociarse, sin discriminaciones raciales, políticas, religiosas, sociales o de género, Por lo tanto, la ley debe acoger de manera explícita este principio a fin de que todos los que tengan las mismas necesidades y deseos de solucionarlas mediante la acción común puedan formar parte de las cooperativas. Esta característica es particularmente definitoria de las organizaciones cooperativas y por ello la legislación debe ser prolija en su tratamiento.

Sentado el principio de la adhesión libre y voluntaria, deben establecerse las consecuencias del ingreso, es decir los deberes y derechos que la calidad de socio trae aparejados. En esta materia las disposiciones deben ser de carácter general, dejando librado a los estatutos de cada cooperativa las precisiones de mayor detalle. Empero, deben quedar legalmente establecidos derechos y deberes tales como la participación de los socios en el gobierno democrático de la cooperativa y el cumplimiento de las obligaciones establecidas en el estatuto.

La cooperativa se propone satisfacer necesidades y aspiraciones de sus socios por medio de una organización común. De allí que la ley deba dejar sentadas con carácter general las obligaciones y derechos que los socios asumen al formar parte de ella.

\section{Régimen económico}

La cooperativa, como organización económica, debe contar con los recursos necesarios para cumplir con el objeto social que se propone en beneficio de sus socios. Esos recursos están constituidos, básicamente, por el capital que aportan los propios socios y por las reservas que se van acumulando como consecuencia de excedentes no distribuidos. Esta materia debe ser objeto de normas específicas que estén consonancia con los principios cooperativos ${ }^{17}$.

Debe tenerse presente que la necesidad de contar con capital suele aconsejar que se otorgue una compensación a los aportes bajo la forma de un interés limitado. También pueden preverse mecanismos de capitalización en proporción con el uso de los servicios de la cooperativa o bajo la forma de fondos rotatorios $u$ otros que posibiliten a la cooperativa contar con recursos adecuados para su desenvolvimiento sin apremios financieros.

17 Si bien la Declaración de Identidad Cooperativa de la $\mathrm{ACl}$ admite la incorporación de capital de terceros existe un amplio debate acerca de su conveniencia (Dante CRACOGNA, «Problemas actuales del derecho cooperativo», Revista de Derecho Privado y Comunitario, N. ${ }^{\circ}$ 2011-3, Buenos Aires, 2012, p. 26 y ss). 
Las reservas formadas con excedentes no distribuidos constituyen un mecanismo de acumulación de recursos financieros que no demandan costos para la cooperativa, por lo que resultan especialmente interesantes para consolidar el patrimonio social. Debe preverse que al menos una parte de tales reservas sean indivisibles, es decir que no resulten apropiables por los socios individualmente sino que integren un auténtico patrimonio de propiedad común.

En todo caso, como las cooperativas deben actuar en igualdad de condiciones con otras organizaciones, deben tener asegurada la posibilidad de acceder a los mecanismos de financiación bancaria y de cualquier otro tipo, de la misma forma que aquéllas.

El excedente económico de la gestión social debe tener previsto en la ley sus posibles destinos, los cuales deben ser decididos por cada cooperativa sea en su estatuto o por resolución de la asamblea. Uno de los tales destinos es la ya referida constitución de reservas. Otro destino puede ser la distribución entre los socios en proporción a las operaciones realizadas con la cooperativa, devolviéndolo así a quienes contribuyeron a su formación (esta devolución también puede realizarse en certificados de aportes de capital) ${ }^{18}$. Finalmente, puede ser dedicado al apoyo de actividades de interés común que la asamblea decida.

Numerosas legislaciones que imponen la obligación de destinar a reserva un cierto porcentaje de los excedentes, lo cual ayuda a consolidar el patrimonio de la cooperativa.

\section{Contabilidad y auditoría}

La ley debe asegurar que los registros contables de las cooperativas sean llevados de manera clara y confiable, tanto en beneficio de los socios como de los terceros que se relacionan con ellas. A tal fin la ley debe contener disposiciones apropiadas, a tono con la capacidad económica y el volumen de operaciones que realicen las cooperativas, sin llegar a exigencias que por su costo o complejidad superen las posibilidades de ellas.

Además de las disposiciones sobre los registros contables, deben existir normas sobre la confección y publicación del balance que es el documento que expresa la situación económico financiera de la coope-

18 Esta es el destino propio y peculiar del excedente en las cooperativas, denominado «retorno». 
rativa ${ }^{19}$. Este documento, además de someterse a la consideración de los socios en asamblea, debe enviarse al registro para conocimiento público.

La auditoría de las cuentas de la cooperativa constituye un recaudo que garantiza su confiabilidad. De allí la conveniencia de establecer que ella sea realizada, sea por profesionales contadores independientes o a través de organizaciones cooperativas especializadas. En este último caso existen las ventajas de la especialización cooperativa y de los menores costos derivados de la organización común.

\section{Los órganos sociales}

La estructura organizativa de la cooperativa es un elemento de gran importancia para su correcto funcionamiento. Ella debe equilibrar la capacidad de gestión ágil y dinámica con el gobierno democrático y el control interno, para lo cual es necesario establecer órganos diferenciados asignando a cada uno de ellos una competencia específica y funciones claramente definidas. Si las funciones se confunden, los distintos órganos interfieren entre sí, surgen conflictos internos y el funcionamiento de la cooperativa se deteriora.

En general existen: un órgano de gobierno (asamblea), un órgano de administración (consejo de administración), y un órgano de control (comité de control). Si bien corresponde al estatuto de cada cooperativa establecer detalladamente cuáles son las funciones de cada uno de tales órganos, la ley debe determinar con carácter general su respectiva competencia, conforme con su naturaleza.

La asamblea - constituida por todos los socios con derecho a un voto cada uno - tiene a su cargo designar a los miembros de los demás órganos y a la auditoría; la aprobación del programa de actividades y del presupuesto; la aprobación del balance anual; además de todas aquéllas cuestiones que, por su importancia, afecten la marcha general de la cooperativa.

El consejo de administración —formado por un número reducido de miembros, por lo general socios - es el encargado de administrar la cooperativa dentro del marco de las disposiciones del estatuto y de las

19 Los registros contables deben adecuarse a la naturaleza de las cooperativas y utilizar una terminología que la refleje. En este sentido es particularmente importante que los aportes realizados por los socios se contabilicen como capital, es decir como parte del patrimonio social, y no cono un pasivo de la manera que pretende la NIC 32 del IASB. 
resoluciones de la asamblea; llevar la contabilidad y someter a la asamblea el balance y el informe anual de sus actividades ${ }^{20}$.

El comité de control o de supervisión - constituido por un número de socios establecido en el estatuto - tiene a su cargo el control de las actividades del consejo de administración con cargo de informar a la asamblea. Puede ser el encargado de designar la auditoría, en lugar de la asamblea.

\section{Disolución y liquidación}

El fin de la existencia de la cooperativa puede producirse por distintos motivos que la ley debe prever expresamente, tales como la decisión de propios socios mediante una mayoría especial; la imposibilidad de cumplir el objeto social; la expiración del período de duración previsto; el incumplimiento de las condiciones establecidas para su registro; etc. Producida la disolución a continuación debe realizarse la liquidación, sea por el mismo consejo de administración o por una comisión especialmente designada al efecto, la cual realizará los activos y pagará el pasivo para luego devolver el capital aportado por los socios, si existiera remanente. Las reservas indivisibles deben tener el destino específicamente previsto por la ley o el estatuto ${ }^{21}$. El comité de supervisión y la auditoría deben vigilar el proceso de liquidación para asegurar su regularidad y transparencia.

En cambio, si la cooperativa se hallara en cesación de pagos la liquidación se hará conforme con el procedimiento previsto en forma general por la legislación sobre quiebras para cualquier organización.

Finalmente, también puede suceder que se produzca la disolución sin liquidación. Tal es el caso de la fusión con otra cooperativa.

Cualquiera que fuese el motivo de la disolución, una vez concluida la liquidación debe procederse a la baja de la cooperativa del registro respectivo, pues concluye entonces su existencia como sujeto de derecho.

\section{Organizaciones de integración y representación}

Uno de los principios cooperativos establece que las cooperativas sirven más efectivamente a sus socios y fortalecen al movimiento coo-

20 Según el modelo de algunas legislaciones, la asamblea designa un consejo de supervisión que, a su vez, designa al comité de dirección.

21 Generalmente suele destinarse a otra organización cooperativa o una entidad de educación o asistencia social. 
perativo trabajando mancomunadamente a través de estructuras locales, nacionales, regionales e internacionales. Por lo tanto, es necesario que la legislación prevea que las cooperativas pueden constituir organizaciones de grado superior para realizar objetivos económicos y de representación ${ }^{22}$.

Tales organizaciones permiten que las cooperativas potencien su capacidad económica en los distintos campos en los que operan, haciendo más eficiente la tarea común merced a los beneficios de escala y al mejor aprovechamiento de los recursos. Por otra parte, en el orden representativo pueden expresar con mayor vigor las aspiraciones del conjunto de las cooperativas llegando así con mayor eficacia a los gobiernos y a la opinión pública en general en la defensa de sus intereses.

Las organizaciones representativas deberían ser reconocidas como interlocutoras de los gobiernos en todas aquellas cuestiones que afectan a las cooperativas y tener participación, siquiera consultiva, en los organismos públicos relacionados con las actividades que ellas desarrollan. Además, deberían ser consultadas cuando se proyecte modificar la legislación específica sobre cooperativas.

\section{La supervisión estatal y el autocontrol}

La supervisión de las cooperativas en cuanto al cumplimiento de las disposiciones legales y reglamentarias que las rigen debe estar, en primer lugar, a cargo de las propias organizaciones cooperativas a través de adecuados mecanismos de autocontrol. De esa manera las cooperativas irán adquiriendo responsabilidad sobre su propia gestión y se evitará la injerencia indebida de los funcionarios públicos en sus asun$\operatorname{tos}^{23}$. La auditoría - ya mencionada - constituye un mecanismo apropiado, si bien no el único.

No obstante, en muchos países existen organismos gubernamentales encargados de la supervisión de las cooperativas. Estos organismos suelen tener simultáneamente a su cargo el registro de las cooperativas. La realización de estas dos actividades (registro y supervisión) por parte de un mismo organismo no parece ofrecer mayores dificultades puesto que no son funciones incompatibles.

22 Dante CRACOGNA, Intercooperacao e integracao cooperativa na America Latina, Pensamento Cooperativo, N. ${ }^{\circ}$ 1, Dezembro 2000, INSCOOP, Lisboa, pp. 111 y ss.

${ }_{23}$ Esta es una materia particularmente sensible, en la que ha de cuidarse celosamente la vigencia del principio de autonomía e independencia de las cooperativas. 
Sin embargo, dichos organismos suelen asimismo cumplir funciones de promoción de las cooperativas, lo cual puede resultar incompatible con las funciones de supervisión y dar lugar a ingerencia excesiva de los funcionarios de gobierno.

\section{La política nacional en materia de cooperativas}

Son numerosos los países que cuentan con un organismo especializado en materia de cooperativas dentro de la administración pública. Tal organismo suele a veces concentrar las tres funciones antes referidas: el registro, la fiscalización pública y la promoción o fomento. La existencia de un único organismo oficial relacionado con las cooperativas puede ofrecer la ventaja de contar con una sola política en materia cooperativa realizada con unidad de criterio, si bien tal concentración de funciones puede al mismo tiempo constituir un riesgo para la autonomía de las cooperativas.

La ubicación de dicho organismo debería estar en un área del gobierno de alcance general o estratégico, como el departamento de planificación o la oficina del primer ministro o del presidente, en lugar de estar en un determinado ministerio. Tal ubicación permite definir e implementar una política única para todas las áreas del gobierno y para toda clase de cooperativas ${ }^{24}$.

En algunos casos la dirección del organismo de cooperativas suele estar a cargo de un directorio formado con representantes del gobierno y del movimiento cooperativo. Esta experiencia ha demostrado buenos resultados al permitir que los representantes de las cooperativas tengan participación en las actividades gubernamentales relacionadas con estas entidades y puedan transmitir sus necesidades y aspiraciones al gobierno. En todo caso, la dirección a cargo de un cuerpo colegiado en lugar de un funcionario único puede contribuir a disminuir el riesgo de las actitudes personales arbitrarias mediante el control grupal. Sin embargo, las decisiones finales que adopte el organismo y que puedan afectar a las cooperativas deben estar sujetas a revisión judicial.

La acción de este organismo resulta particularmente importante puesto que ella pone en juego el principio de autonomía e independencia de las cooperativas, expresamente incluido dentro de la Declaración de Identidad Cooperativa aprobada por la $\mathrm{ACl}$ en el Congreso del Centenario. Dicho principio puede verse afectado tanto por la intromisión indebida del gobierno en las cooperativas como por un paterna-

24 Anexo Res. 56/114 ONU. 
lismo que las convierta en entidades dependientes del gobierno. Radica aquí una cuestión crucial para el genuino desarrollo cooperativo pues en muchas ocasiones la acción promocional del gobierno ha llegado a sustituir la acción de los propios interesados, convirtiendo de esa manera a las cooperativas en verdaderos apéndices de las políticas oficiales o en meros instrumentos para su realización ${ }^{25}$.

\section{$\mathrm{XV}$. Los conflictos en las cooperativas y su solución}

En las cooperativas, como en toda organización humana, la presencia de los conflictos es algo natural. Estos pueden plantearse entre la cooperativa y los socios o entre los socios o entre las cooperativas. Lo importante es que los conflictos tengan cauces adecuados de solución a fin de que puedan superarse de manera rápida y económica.

Un método de solución de conflictos implementado con éxito en distintos países consiste en el arbitraje, generalmente a cargo de árbitros pertenecientes a organizaciones especializadas o a las propias organizaciones de integración cooperativa. El arbitraje tiene la ventaja de su bajo costo, sencillez y relativa rapidez. Lo mismo puede decirse de la mediación.

Cuando los conflictos no pueden resolverse por medios libremente aceptados por las partes involucradas, éstas pueden acudir a la justicia, instancia que debe estar disponible al igual que para cualquier otra persona u organización. En tales casos parece conveniente que intervengan los tribunales ordinarios, sin necesidad de que exista un fuero especial para los asuntos de cooperativas.

\section{$\mathrm{XVI}$. Aspectos fiscales}

Una cuestión delicada y de especial importancia es la relativa al tratamiento impositivo de las cooperativas; es decir qué tributos deben pagar estas entidades y si deben ser tratadas de manera diferente al resto de las organizaciones económicas.

Acerca de este tema existen distintas opiniones: por una parte, hay quienes sostienen que las cooperativas deben estar eximidas de tributación por cuanto ellas contribuyen al desarrollo económico y social de la comunidad y, por otra, hay quienes afirman que deben tener exacta-

25 Sostenía Alexander Laidlaw que «es muy común que el fuerte abrazo gubernamental termine siendo un factor de fracaso para las cooperativas» («Cooperatives in the Year 2000», ICA XXVII Congress Moscow 1980, ICA, London, 1980, p. 161). 
mente el mismo tratamiento que las demás entidades, sin ninguna diferencia. Entre medio están los que sostienen que deben estar exentas de impuestos durante una cierta etapa inicial o bien según el volumen o la clase de actividad que realizan.

Lo que resulta indiscutible es que las cooperativas no deben subsistir gracias a un tratamiento fiscal de preferencia ya que esa situación las hace vivir artificialmente y les impide alcanzar su plena autonomía pues siempre dependerán del favor gubernamental.

Por otra parte, lo dicho no significa que las cooperativas deban pagar los mismos impuestos que las empresas lucrativas comunes, puesto que son organizaciones diferentes. En todo caso, el tratamiento tributario que se les otorgue debe ser conforme con su particular naturaleza de entidades fundadas en el esfuerzo propio y la ayuda mutua para prestar servicios a sus socios. De allí que puedan estar sujetos a determinados tributos y no a otros.

Es verdad que los sistemas impositivos nacionales difieren grandemente entre sí, por lo que toda generalización resulta difícil y riesgosa. Pero la línea orientadora para definir el tratamiento impositivo debe ser la peculiar naturaleza de las cooperativas, tal como se halla definida en la Declaración de Identidad Cooperativa de la $\mathrm{ACl}$. De ella surge que en las cooperativas el excedente no se reparte en proporción al capital aportado; que todos los socios cuentan con un voto; que existen reservas irrepartibles; que los excedentes se devuelven a quienes los formaron o bien se destinan a fines de interés común; etc. Todos esos rasgos configuran una organización diferente de las empresas lucrativas comunes y el régimen tributario debe tratarlas conforme con esa particular naturaleza.

\section{Consideraciones finales}

A manera de conclusión cabe señalar algunos aspectos generales a tener en cuenta en relación con el tema tratado:

Es conveniente que la Constitución —como máximo nivel del ordenamiento jurídico nacional- dé cabida a alguna disposición que reconozca a las cooperativas y les garantice un tratamiento legislativo acorde con sus peculiares características.

La elaboración de la legislación cooperativa debe realizarse con la activa participación de sus destinatarios, es decir las propias organizaciones del movimiento cooperativo ${ }^{26}$, como manera de asegurar que ella responda a las genuinas características y necesidades de las cooperativas.

26 Recomendación 193 OIT, Cap. III, 10. 
La experiencia legislativa de países con mayor desarrollo cooperativo como así también los documentos elaborados por los organismos internacionales y los proyectos de armonización legislativa realizados a nivel regional e internacional deben ser adecuadamente tomados en cuenta como elementos de orientación. ${ }^{27}$

La legislación cooperativa, como así también toda otra disposición legislativa, debe otorgar a las cooperativas un trato cuando menos igual al que se confiere a organizaciones de otra naturaleza, especialmente a las sociedades comerciales, pero sin asimilarlas indebidamente con éstas como a veces se sostiene con fundamento en una pretendida defensa de la competencia. ${ }^{28}$

La autonomía de las cooperativas con relación al Estado constituye un requisito que la ley debe reconocer de manera explícita y sin restricciones a todas las cooperativas, cualquiera sea su actividad específica.

La redacción y el lenguaje utilizados por la legislación cooperativa deben ser adecuados a la comprensión de la mayor cantidad posible de la población y, especialmente, de los potenciales miembros de las cooperativas. Todo ello en la medida compatible con las exigencias técnicas propias del lenguaje legal.

Finalmente, es conveniente dar una amplia difusión a la legislación cooperativa a fin de que todos los sectores de la población conozcan esta forma de organización a la que pueden recurrir para encarar la solución de sus necesidades económicas y sociales.

27 Hagen Henrÿ sostiene que la Declaración de Identidad Cooperativa de la ACl, los Lineamientos de la Res. 56/114 de la ONU y la Recomendación 193 de la OIT constituyen en núcleo del Derecho Público Internacional Cooperativo, el cual debe ser respetado cuando se legisla en materia cooperativa (Guidelines, cit., p. 5).

28 Dante CRACOGNA, «La legislación cooperativa en el Siglo XXI», Cooperativismo y Desarrollo, N. ${ }^{\circ}$ 100, Bogotá, 2012, p. 180. 